\title{
GPPS-BJ-2019-161
}

\section{Piezoelectric Wave-mode Transducers for Structural Health Monitoring of Joint Structures in Cylinders}

\author{
Lin $\mathbf{L I}^{1,2}, \quad$ Wenjun WANG ${ }^{1}, \quad$ Yu FAN ${ }^{1,2^{*}}$ \\ 1 School of Energy and Power Engineering, Beihang University, Beijing 100191, China \\ ${ }^{2}$ Beijing Key Laboratory of Aero-engine Structure and Strength, Beijing 100191, China
}

\begin{abstract}
Joint structures, such as riveting, hinges and flanges, are widely used in complex mechanical systems. A small unexpected change of the connection status may lead to wear, leakage, or other severe safety consequences especially in the aerospace field. The transmission and conversion of the guided waves can be used to quantify the variation of connection status of the joint structures. This gives rise to the challenge of exciting and sensing only one specific wave mode. In this paper, wave-mode piezoelectric transducers were designed for the torsional, longitudinal and flexural waves in cylinder structures respectively. The idea was to use the distribution and interconnection of the piezoelectric materials to cancel the charge contributed from the undesired waves. We conducted numerical simulations to demonstrate the selective coupling features of the designed wave transducers and found several orders of magnitude difference in voltages between targeting wave mode and other wave modes. These wave transducers were then extended to monitor the connection status of joint structure and results showed the transducers can detect very small amount of wave mode conversion due to local damages of the joint structures.
\end{abstract}

\section{INTRODUCTION}

Large-scale mechanical systems such as spacecraft contain thousands of simple components connected by joint structures. Due to complex operating condition, these structures undergo contact surface oxidation, thermal deformation, preload reduction and other minor local changes, which are able to effect the whole dynamic properties of mechanical systems and even invalidate systems (Peairs, Park and Inman, 2004). Structural health monitoring (SHM) for joint structures is therefore crucial to ensure structural safety.

Three types of SHM systems were successively developed in last two decades: (1) vibration based SHM; (2) SHM based on electro-mechanical impedance; and (3) guidedwave based SHM. The vibration based SHM techniques (M
Maia, 2006) (Deraemaeker et al., 2010) identified local minor damage by comparing changes in natural frequencies and dynamic responses before and after occurrence of damage. Since the local damage of joint structure commonly cause limited changes in the overall dynamics, sensitivity of this method may be unsatisfactory for joint structures. Alternatively, by piezoelectric transducers attached to the monitored portion, electrical impedance of piezo-materials was verified to be reasonably sensitive to the status of monitored site (Hamzeloo, Shamshirsaz and Rezaei, 2012). This method was then extended to identify the status of a single bolt (Farrar et al., 2009). Nevertheless, SHM techniques based on electro-mechanical impedance (Na and Baek, 2018) have the same limitation of the vibration based ones for they both used the steady-state dynamic characteristics.

The probably most popular candidate of SHM is guided wave based SHM technique, which has attracted substantial attention in the past two decades. It has several inherent advantages: (1) small wavelength ensures a sufficient interaction of guided waves and local minor damage; (2) high excitation frequency of guided wave is far from operating frequency and ambient frequency, which barely perturb the transmission of guided waves; (3) The characteristics of piezoelectric materials make guided waves possess the capability of a wide sweep range. For these reasons, we develop wave based approaches for the SHM of joint structures in this work.

In wave based SHM approaches, the transducers are respectively arranged at two ends of the structure in general. One is to actuate guided waves and the other one is to receive the excited guided waves. Features of guided wave, such as velocity, attenuation, scattering, and mode conversion, are used to indicate changes in structural dynamics. Lee (Lee and Staszewski, 2003) simulated the interaction between lamb wave and damage in metallic plate. The study found that damage can cause transmission, reflection and mode conversion (from A0 to S0) of lamb waves. Fromme

* Please address all correspondence to this author (Y. Fan, E-mail: fanyu04@buaa.edu.cn). 
(Fromme, 2004) analysed the wave mode conversion when lamb wave transmit through a circular damage hole. The results show that the amplitude of $\mathrm{S} 0$ mode converted from A0 mode has a strong correlation with the depth of the hole. Willberg (Willberg et al., 2012) investigated laminated composited plates that are widely used in aerospace industry and demonstrated that continuous mode transitions occur due to the asymmetry of layered materials. Li (Li et al., 2015) also demonstrated that slight asymmetry can lead to wave mode conversion in cylindrical shell.

The aforementioned literature shows that wave mode conversion characteristics can be a promising indicator to monitor slight status changes of the joint structures. However, in practice the measurement of the conversion among waves is a challenging task, especially when there are many travelling waves (e.g. in cylinders). Furusawa (Furusawa, Kojima and Morikawa, 2015) designed a circumferentially distributed electromagnetic transducer for the longitudinal and torsional waves in cylinders. Kharrat (Kharrat et al., 2014) presented circumferentially distributed piezoelectric actuators for the first order torsional wave in cylindrical shells. While the above-mentioned literatures have designed transducers that can actuate certain cylindrical waves, a general design approach for all types and orders of wave is lack. Moreover, verification has not been conducted to guarantee the excited guided wave is single mode, which limits the accuracy detected wave mode conversion.

In this paper, a design approach of piezoelectric wavemode transducers is proposed for cylinders. The method is applicable for both low-order and high-order torsional, longitudinal and flexural waves. The designed wave-mode transducer is only coupled with the targeting wave, and has very low interaction with the other waves. This strong selective coupling behaviour is achieved by setting different polarization directions and distributions of piezoelectric materials according to the orthogonality of waves. Numerical studies are conducted to verify such features of the designed transducers. These transducers are then applied to detect the wave mode transmission and conversion in a drum-flange structure. Equivalent thin layer method(Yao, Wang and Zhai, 2015) is used to model the flange. The change of whole joint status and local damage are simulated by elastic modulus of equivalent thin layer and local elements respectively. The relationship and sensitivity between the transducers' output with the local damage of the flange are studied.

\section{Design of wave-mode piezoelectric transducers}

\subsection{Wave propagation in cylinders}

It is well known that the guided waves in cylindrical are multi-mode and dispersive in frequency. To design a transducer only couples with one particular wave, the modal shape and dispersion relation of the guided waves should be known in prior. To do this normally the cylindrical coordinate system is employed, as shown in Figure 1, where $\omega, \xi$, and $\eta$ are radial, circumferential and axial displacements, respectively. Term $R$ is the radius of cylinder, or the distance from the centre of shell to the mid-plane of the shell.
Term $h$ is the thickness of shell. The rotational angle is $\varphi$. The differential equations governing the propagation of waves on cylinders (Nilsson and Liu, 2015) are as follow:

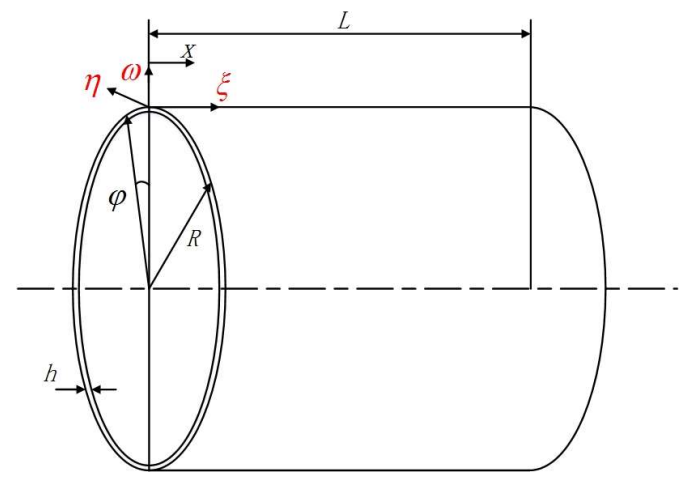

Figure 1 Coordinates and displacement of cylinder

$$
\left\{\begin{array}{l}
\frac{\partial^{2} \xi}{\partial x^{2}}+\frac{1-v}{2 R^{2}} \frac{\partial^{2} \xi}{\partial \varphi^{2}}-\frac{1-v^{2}}{E h} \mu \ddot{\xi}+\frac{1+v}{2 R} \frac{\partial^{2} \eta}{\partial x \partial \varphi}+\frac{v}{R} \frac{\partial \omega}{\partial x}=0 \\
\frac{1+v}{2 R} \frac{\partial^{2} \xi}{\partial x \partial \varphi}+\frac{1-v}{2} \frac{\partial^{2} \eta}{\partial x^{2}}+\frac{1}{R^{2}} \frac{\partial^{2} \eta}{\partial \varphi^{2}}-\frac{1-v^{2}}{E h} \mu \ddot{\eta}+\frac{1}{R^{2}} \frac{\partial \omega}{\partial \varphi}=0 \\
\frac{v}{R} \frac{\partial \xi}{\partial x}+\frac{1}{R^{2}} \frac{\partial \eta}{\partial \varphi}+\frac{\omega}{R^{2}}+\frac{h^{2}}{12}\left(\frac{\partial^{4} \omega}{\partial x^{2}}+\frac{1}{R^{2}} \frac{\partial^{4} \omega}{\partial \varphi^{4}}+\frac{2}{R^{2}} \frac{\partial^{4} \omega}{\partial x^{2} \partial \varphi^{2}}\right) \\
+\frac{\mu\left(1-v^{2}\right)}{E h} \ddot{\omega}=\frac{p\left(1-v^{2}\right)}{E h}
\end{array}\right.
$$

where $E$ is the Young's modulus, $v$ is Poisson's ratio, and $\mu$ is the mass per unit area. $\dot{\omega}$ and $\ddot{\omega}$ indicate derivative with respect to time.

For a wave propagating along the positive $\mathrm{x}$-axis, displacements of three directions can be written as:

$$
\begin{aligned}
& \omega(x, \varphi, t)=\sum_{n} A_{n} \cos (n \varphi) \cdot \exp \left[i\left(\Omega t-\lambda_{n} x\right)\right] \\
& \xi(x, \varphi, t)=\sum_{n} i B_{n} \cos (n \varphi) \cdot \exp \left[i\left(\Omega t-\lambda_{n} x\right)\right] \\
& \eta(x, \varphi, t)=\sum_{n} C_{n} \sin (n \varphi) \cdot \exp \left[i\left(\Omega t-\lambda_{n} x\right)\right]
\end{aligned}
$$

where $n$ is the wave number along circumferential direction, $\lambda_{n}$ is the wave number along axial direction, and $\Omega$ is angular frequency. $A_{n}, B_{n}$, and $C_{n}$ are the displacement amplitudes. $B_{n}$ is multiplied by $i$ to avoid complex numbers in the equations resulting from inserting Eq. (2) in Eq. (1). For free vibration, $p=0$ in the last equation of Eq. (1). Equation (2) is substituted into Eq. (1) and this yields a system of equations written as:

$$
[Q] \cdot\left\{\begin{array}{l}
A_{n} \\
B_{n} \\
C_{n}
\end{array}\right\}=\left\{\begin{array}{l}
0 \\
0 \\
0
\end{array}\right\}
$$

where the elements of the symmetric matrix $[Q]$ are: 


$$
\left\{\begin{array}{l}
Q_{11}=\frac{1}{R^{2}}+\frac{h^{2}}{12}\left(\lambda_{n}^{4}+\frac{n^{4}}{R^{4}}+\frac{2 n^{2} \lambda_{n}^{2}}{R^{2}}\right)-\frac{\mu \Omega^{2}\left(1-v^{2}\right)}{E h} \\
Q_{22}=\lambda_{n}^{2}+\frac{\left(1-v^{2}\right) n^{2}}{2 R^{2}}-\frac{\Omega^{2} \mu\left(1-v^{2}\right)}{E h} \\
Q_{33}=\frac{n^{2}}{R^{2}}+\frac{\left(1-v^{2}\right) \lambda_{n}^{2}}{2}-\frac{\left(1-v^{2}\right) \mu \Omega^{2}}{E h} \\
Q_{12}=\frac{v \lambda_{n}}{R}=Q_{21} \\
Q_{13}=\frac{n}{R^{2}}=Q_{31} \\
Q_{23}=\frac{n \lambda_{n}(1+v)}{2 R}=Q_{32}
\end{array}\right.
$$

The dispersion relations of the waves are obtained by solving problem of $\operatorname{Det}[Q]=0$, namely:

$$
K_{4} \lambda_{n}^{8}+K_{3} \lambda_{n}^{6}+K_{2} \lambda_{n}^{4}+K_{1} \lambda_{n}^{2}+K_{0}=0
$$

where $K_{0}$ to $K_{4}$ are constant coefficients. For each given angular frequency $\Omega$ and circumferential wavenumber $n$, there are eight solutions of $\lambda_{n}$ or rather four pairs of solutions. Each pair represents waves travelling in both the positive and negative directions. If $\lambda_{n}^{2}$ is a negative value, then $\lambda_{n}$ is an imaginary number, and this represents an evanescent wave. Otherwise a positive $\lambda_{n}^{2}$ represents a propagating wave. Note that the associated eigenvector contains three terms, $A_{n}, B_{n}$, and $C_{n}$, representing the proportions of longitudinal, torsional and flexural deformation within a wave mode.

The material of the cylinder was chosen as steel. Elastic modulus, Poisson's ratio, and density are respectively $2 \mathrm{e}+11 \mathrm{~N} / \mathrm{m}^{2}, 0.3$, and $7.8 \mathrm{~kg} / \mathrm{m}^{3}$. Radius and thickness of cylinder are $0.5 \mathrm{~m}$ and $0.003 \mathrm{~mm}$. The dispersive curves are shown in Figure 2. The frequency range is $0-20 \mathrm{kHz}$ and the calculated circumferential wavenumber range is $0-12$. The proportions of the three basic deformation are illustrated by the depth of colour. It is observed that as frequency increases, the wave-shapes quickly evolves into longitudinal, torsional or flexural modes. Depending on the final dominant deformation, the three groups of waves in Figure 2 are respectively distinguished as longitudinal, torsional and flexural waves. These three wave modes are abbreviated as $\mathrm{L}(n), \mathrm{T}(n)$ and $\mathrm{F}(n)$. The group velocity (shown in Figure $3)$ is then obtained by:

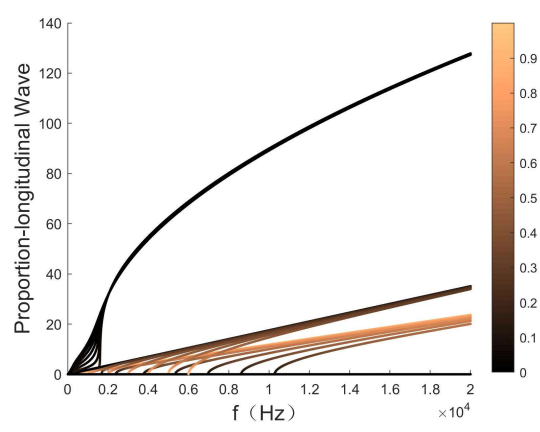

(a)

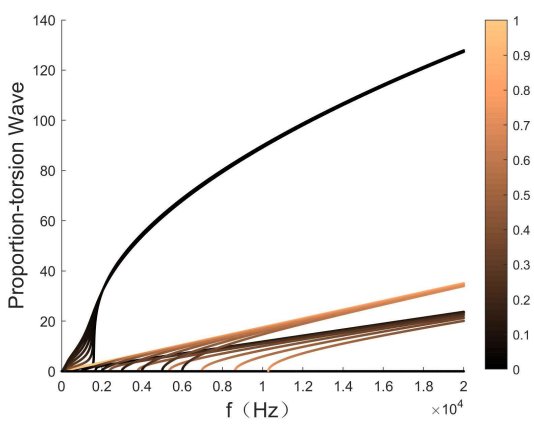

(b)

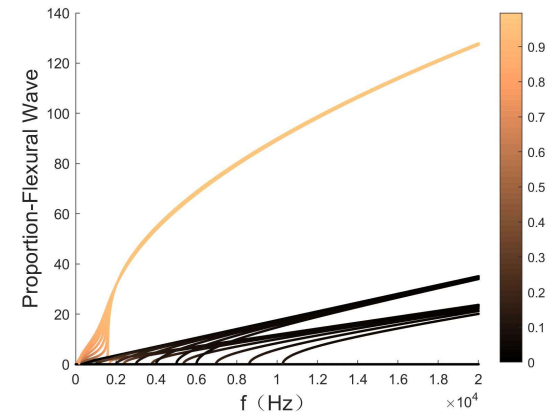

(c)

Figure 2 Dispersion curves of wave mode in cylinder. The colour represents the proportion of each coefficient $(A, B$, and $C)$ in the wave mode. The lighter the colour, the higher the proportion. The three groups of curves can then be identified as (a) longitudinal, (b) torsional and (c) flexural wave.

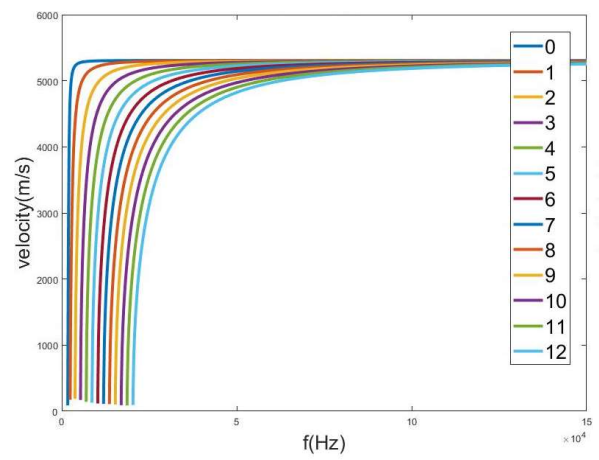

(a)

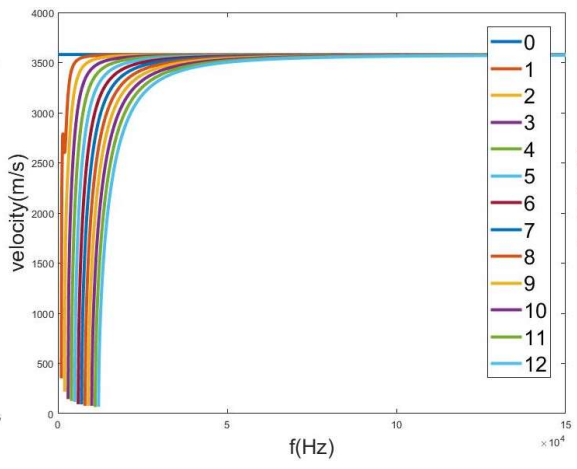

(b)

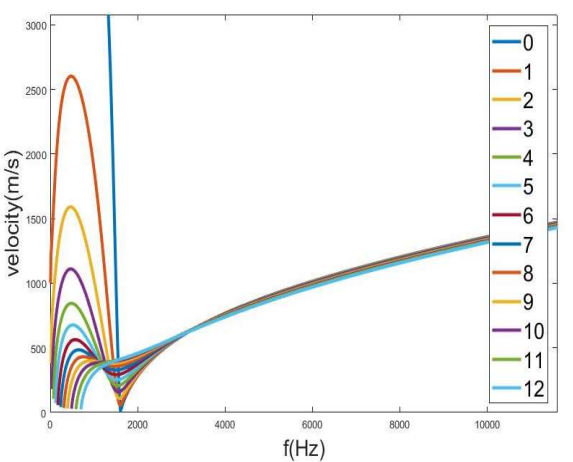

(c)

Figure 3 The group velocity of (a) longitudinal (b) torsional and (c) flexural wave. The number listed in legend is circumferential wavenumber $n$. 


$$
c_{g}=\frac{\partial \bar{\omega}}{\partial \lambda}
$$

The group velocities of longitudinal and torsional wave undergo sharp initial increase and finally converge to phase velocity. This is in accordance with aforementioned evolution of waveform proportion and also illustrate the non-dispersive characteristic of the both. On the contrary, the dispersive feature of flexural wave can be observed that all curves are eventually diverge. Group velocity will be used later to accurately predict the arrival time of guided wave.

\section{2 designing the piezoelectric transducer}

The desired piezoelectric transducers are the ones that can only couple with one wave. Consequently, we can use them to extract the weight of such a wave mode from the overall displacement field of a cylinder, which is the weighted superposition of all the waves:

$$
\vec{u}=\left[\begin{array}{c}
\omega \\
\xi \\
\eta
\end{array}\right]=\sum_{n=0}^{\infty} a_{n} \vec{\Psi}_{L, n}+b_{n} \vec{\Psi}_{T, n}+c_{n} \vec{\Psi}_{F, n}
$$

where $\vec{u}$ is the displacement field, $a, b, c$ are the weight factors, and $\vec{\Psi}$ is the wave mode shape. The voltage response of the piezoelectric transducer is:

$$
V(\vec{u})=\sum_{n=0}^{\infty} a_{n} v\left(\vec{\Psi}_{L, n}\right)+b_{n} v\left(\vec{\Psi}_{T, n}\right)+c_{n} v\left(\vec{\Psi}_{F, n}\right)
$$

where $v$ indicates the voltage induced by $\mathrm{L}(n), \mathrm{T}(n)$, and $\mathrm{F}(n)$. Through appropriate distribution and interconnection of the piezoelectric materials, function $v()$ can only yield significant value only for one wave yet return minor values for the other waves.

Let us first discuss the design of transducers for the firstorder longitudinal wave. In this case, the polarization direction of piezoelectric materials is parallel to the radial direction, as shown in Figure 4(a). The outer and inner surfaces are

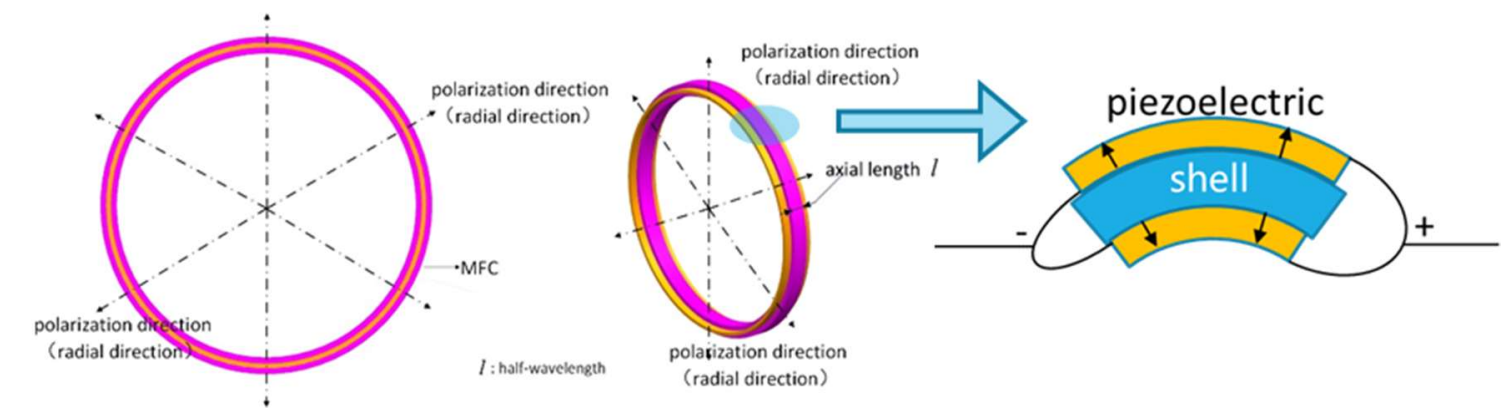

(a)

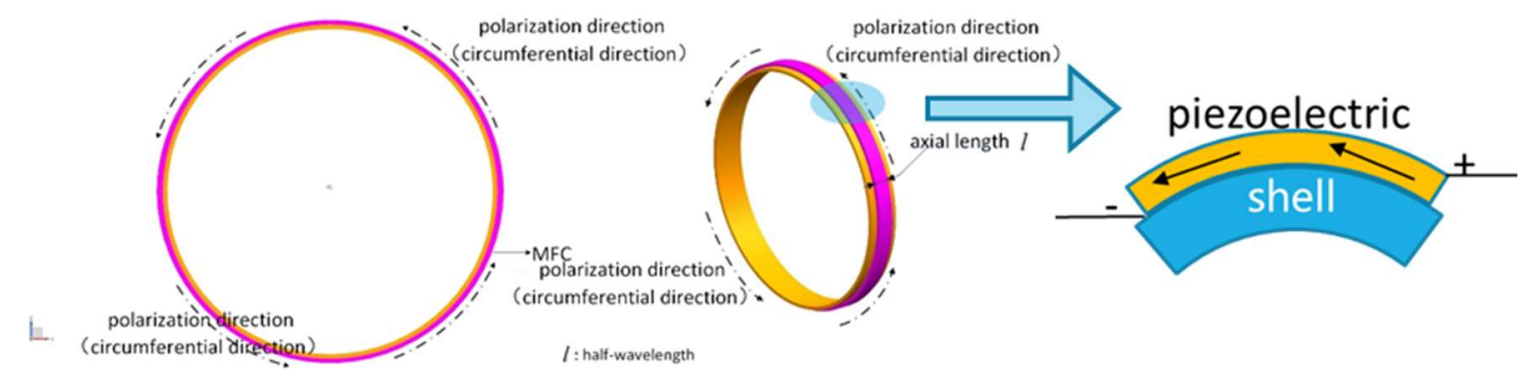

(b)
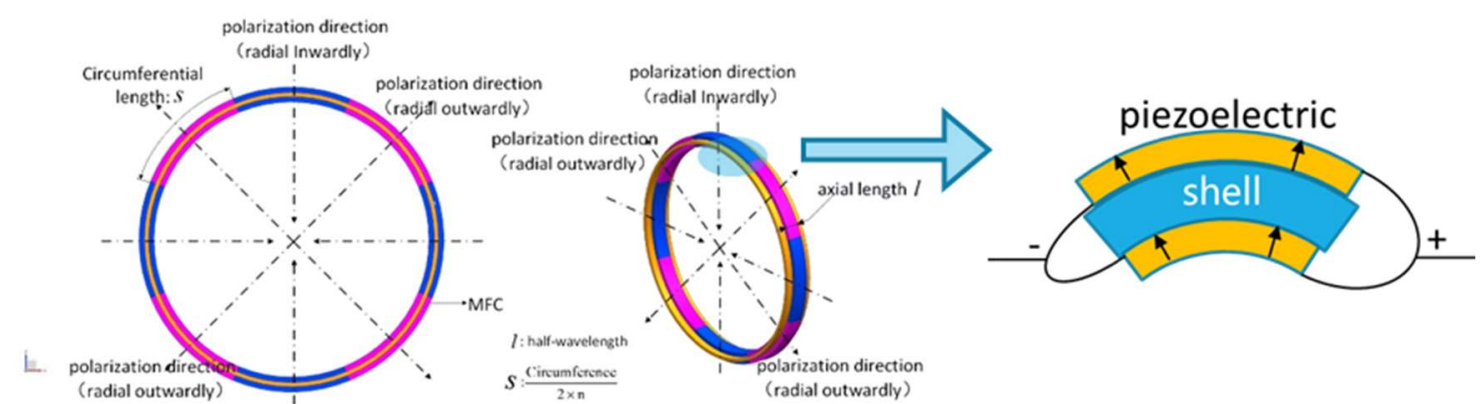

(c)

Figure 4 Design schemes including polarization direction, distributed function, parallel piezoelectric patches, shunting circuit, and axial length for (a): $L(0),(b)$ : $T(0)$, and (c): $F(4)$. 


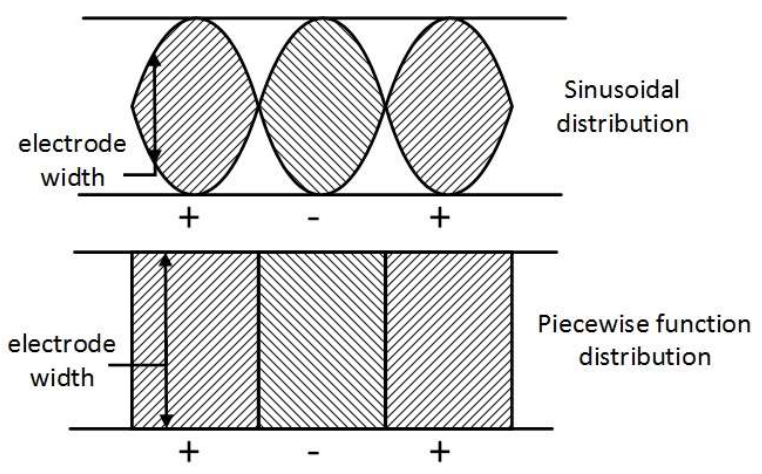

Figure 5 electrode width of piezoelectric transducer array along axial direction

connected to the positive and negative output ends of the transducer respectively. The voltage induced by a longitudinal wave can be written as:

$$
V_{r}=\frac{Q_{r}}{C}=\frac{1}{C} \iint_{s} D_{r} d s
$$

where $Q_{r}$ represents electricity quantity, $C$ is the intrinsic capacitance, $D_{r}$ is electric displacement in the radial direction and $s$ is the area of the piezoelectric material at one radius. The final expression of $V_{r}$ is:

$$
V_{r}=\sum_{n=0}^{\infty} \kappa \int_{0}^{2 \pi} \Delta(\varphi) d_{31} \varepsilon(\varphi) d \varphi
$$

where $\kappa$ is a constant value associated with each wave mode, $\Delta(\varphi)$ is the length of piezoelectric along axial direction, and $\varepsilon(\varphi)=A_{\varepsilon} \cos (n \varphi)$ is strain field induced by the passage of wave with circumferential wave number $n$.

For a given longitudinal wave, i.e. $n$ is fixed, if the length function also follows the form of the cosine function, i.e. $L(\varphi)=L_{0} \cos (m \varphi)$, it can be proved that $V_{r}$ is non-zero only when $n=m$ by the orthogonal relations of the cosine functions. This implies that the length function of the piezoelectric materials acts like a filter function that endows the transducer with the selectively coupling capacity with the desired longitudinal wave (the one with circumferential wave number $n=m$ ). Figure 4(a) shows the transducer for the first-order longitudinal wave, where only a constant length is required. Note that the torsional wave modes induce zero voltage for the longitudinal transducers, due to the polarization direction. The voltage induced by the flexural waves is cancelled by the collocated piezoelectric materials in the outer and inner races. For these reasons, we can prove that the transducer with the configuration shown in Figure 4(a) is only coupled with the first-order longitudinal wave.

This procedure is also applied when designing transducers for $\mathrm{T}(n)$ and $\mathrm{F}(n)$ waves. The transducer for the first-order torsional wave is shown in Figure 4(b), where the poling is along the circumferential direction. As for flexural waves, the poling is also along the radius direction, except that the poling of collocated piezoelectric materials different from the longitudinal case. This is to filter out the contribution of the longitudinal waves of the transducer output. Waves $F(4)$ and $\mathrm{F}(6)$ are considered, their associated length functions are shown in Figure 5. Nevertheless, sinusoidal distribution is hard to implement for both simulation and experiment. Therefore, we use piecewise distribution as an approximation:

$$
L(\varphi)=\left\{\begin{array}{l}
A_{F} \quad \frac{2 i \pi}{n} \leq \varphi<\frac{(2 i+1) \pi}{n}, i=0,1,2, \ldots, n-1 \\
-A_{F} \quad \frac{(2 i-1) \pi}{n} \leq \varphi<\frac{2 i \pi}{n}, i=1,2,3, \ldots, n
\end{array}\right.
$$

As show in Figure 5, the signs of positive and negative represent radial outward and inward direction respectively. This scheme is more feasible and ensures orthogonality with guided wave mode as well.

In wave-based SHM, the wavelength should be at the same magnitude of the targeting singularities to ensure sufficient wave scattering. On the other hand, to actuate one wave, the external force should be applied in a suitable area, which in this paper corresponds to the length of the transducer. In this work, the wavelength of the actuated wave is twice the length of joint structure; and the length of the piezoelectric transducer equals to half-wavelength. Then the working frequency of transducers can be known by

$$
2 A f=c_{g}
$$

where $A$ is axial length of joint structure.

\section{Verification of the wave mode transducers}

The transducers for waves $\mathrm{L}(0), \mathrm{T}(0), \mathrm{F}(6)$, and $\mathrm{F}(4)$ are modelled and analysed by ANSYS, where the piezoelectric materials are modelled by SOLID5 elements, and the host cylinder is modelled by SHELL181 elements. The material of

Table 1 Flexibility coefficients of PZT5H

\begin{tabular}{c|ccccccccc}
\hline & $S_{11}$ & $s_{12}$ & $S_{13}$ & $s_{22}$ & $s_{23}$ & $S_{33}$ & $s_{44}$ & $s_{55}$ & $s_{66}$ \\
\hline $\mathbf{P} 5 \mathbf{h}\left(10^{10} \mathrm{~N} / \mathrm{m}^{2}\right)$ & $\mathbf{1 2 . 6}$ & $\mathbf{7 . 9 5}$ & $\mathbf{8 . 4 1}$ & $\mathbf{1 2 . 6}$ & $\mathbf{8 . 4 1}$ & $\mathbf{1 1 . 7}$ & $\mathbf{2 . 3}$ & $\mathbf{2 . 3}$ & $\mathbf{2 . 3 5}$ \\
\hline
\end{tabular}

Table 2 Piezoelectric strain constants of PZT5H

\begin{tabular}{c|ccc}
\hline & $d_{31}$ & $d_{33}$ & $d_{15}$ \\
\hline $\mathbf{P} 5 \mathbf{h}(\mathrm{m} / \mathrm{V})$ & $\mathbf{1 2 . 6}$ & $\mathbf{8 . 4 1}$ & $\mathbf{1 2 . 6}$ \\
\hline
\end{tabular}

Table 3 Permittivity of PZT5H $\left(e_{0}=8.854 \times 10^{-12} \mathrm{~F} / \mathrm{M}\right)$

\begin{tabular}{c|ccc}
\hline & $e_{11}$ & $e_{22}$ & $e_{33}$ \\
\hline $\mathbf{P 5 h}$ & $\mathbf{1 7 0 0}$ & $\mathbf{1 7 0 0}$ & $\mathbf{1 4 7 0}$ \\
\hline
\end{tabular}


piezoelectric element and shell element are piezoelectric ceramic PZT5H and steel respectively. The material parameters of PZT5H are shown in Table 1, Table 2, and Table 3.

The voltage DOFs of piezoelectric transducer array on different radii are coupled to be identical to simulate connection patterns in Figure 4. Three-cycle sinusoidal voltage signal with sinusoidal window function written as:

$$
V=100 \sin (3 \cdot 2 \pi f t) \sin (2 \pi f t)
$$

and shown in Figure 6(b) is applied to positive pole and employed as stimulant signal. The voltage of negative pole is set to zero to simulate ground.

Due to assumptions made regarding the displacement field in thickness direction, ' $f * h$ ' problem arises when $f$ increases to be large enough. Since guided wave propagation in thickness direction cannot be modelled accurately, stiffening effects may be encountered, resulting in locking and overestimation of wave velocity. Therefore, wave velocity examination is employed after every wave propagation simulation to prevent effect of these restrictions. This examination is conducted by setting the distance between actuating point and sensing point to five wavelengths.

Firstly, we verified the actuation capacity of the transducers by imposing voltage on the transducer and observing the resulting displacement field. Results show that the transducers merely actuate pre-setting guided wave mode. An example is shown in Figure 6(c), where the displacement field is actuated by a transducer designed for $F(6)$ wave.
Secondly, we verified the sensing capacity of the transducers by applying the external force to the host cylinder and observing the transducer output. To test the $\mathrm{L}(0)$ and $\mathrm{T}(0)$ transducers, the forces are applied at the left end by evenly distributed sinusoidal axial force and circumferential force along circumference respectively. To test the $\mathrm{F}(6)$ and $\mathrm{F}(4)$ transducers, the forces applied to the left end write:

$$
F=\sin (n \theta) \sin (2 \pi f \cdot t)
$$

where $\theta$ is the angle of node in cylindrical coordinate system. The working frequencies for $\mathrm{L}(0), \mathrm{T}(0), \mathrm{F}(6)$ and $\mathrm{F}(4)$ are $26.54 \mathrm{khz}, 17.90 \mathrm{hz}, 1.47 \mathrm{khz}$, and $1.34 \mathrm{khz}$ selected from the procedure in section 2.2. The result of $\mathrm{L}(0)$ transducer array is shown in Figure 7 . The time of silence area is approximately equal to five period and accords with wave velocity examination. The voltage induced by $\mathrm{L}(0)$ is $10^{0} \mathrm{~V}$ while by $\mathrm{T}(0), \mathrm{F}(6)$ and $\mathrm{F}(4)$ are $10^{-11}, 10^{-8}$, and $10^{-8} \mathrm{~V}$ respectively. When $\mathrm{T}(0), \mathrm{F}(6)$, and $\mathrm{F}(4)$ transducer arrays are successively implemented, the exponential gap can also be observed between voltages induced by these four wave modes. This indicates that piezoelectric transducer array can only sense the targeting wave mode.

The third verification is then conducted by replacing force with piezoelectric actuator, in order to test the actuatordamage-sensor interaction, as shown in Figure 8. The left end and right end extend 5 wavelengths to prevent interference from reflected waves. The working frequencies for sensor

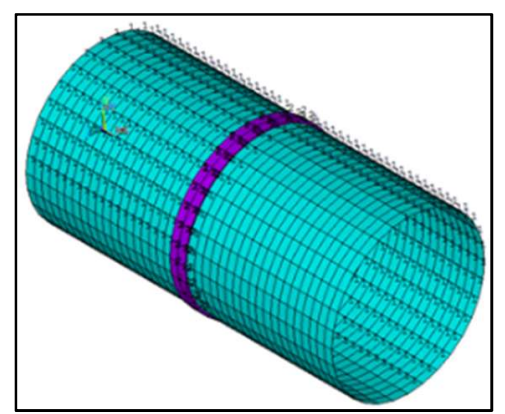

(a)

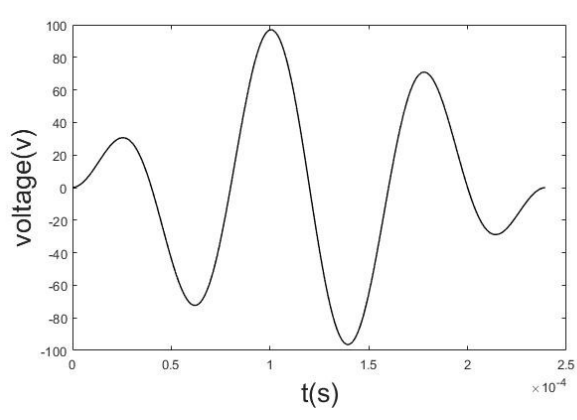

(b)

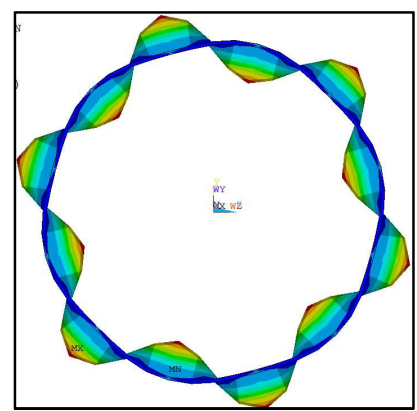

(c)

Figure 6 (a) FE model for the test of a single piezoelectric transducer, (b) external voltage applied to the transducers, and (c) displacement field actuated by the $F(6)$ transducer
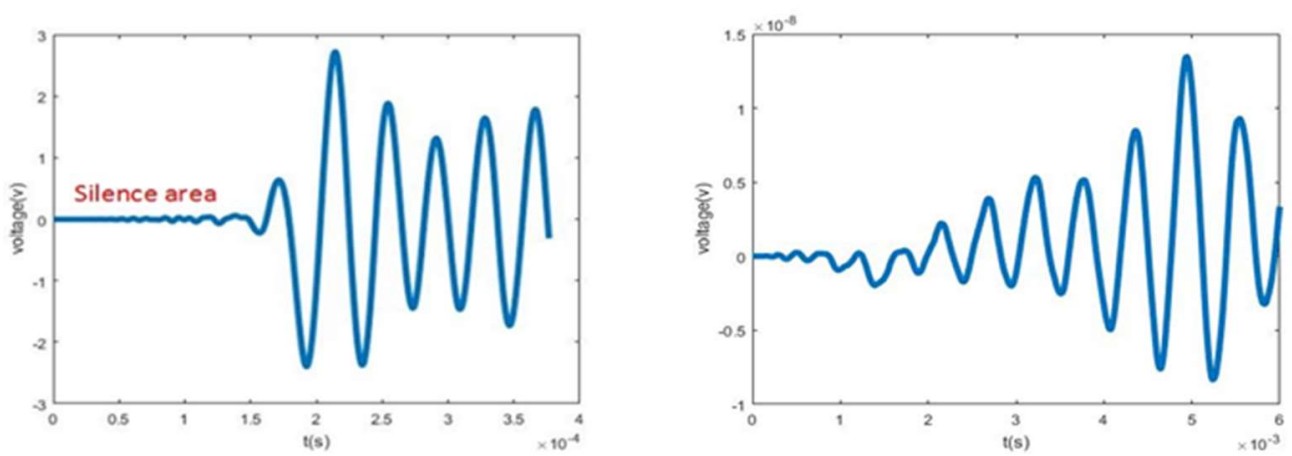

Figure 7 Sensor output of the $L(0)$ wave excited by : (a) force of $L(0)$, (b) force of $F(6)$ 


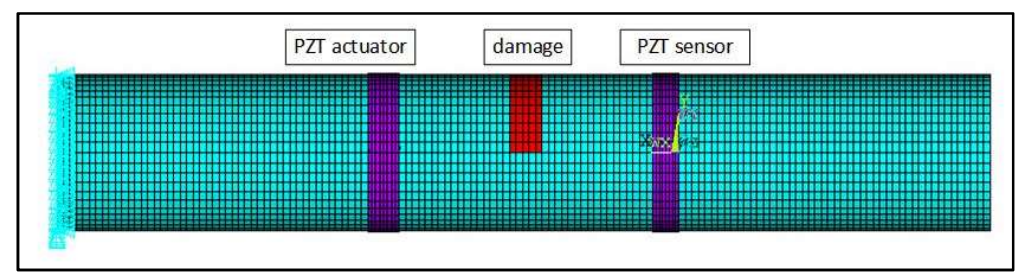

Figure $8 \mathrm{FE}$ model for the test of two piezoelectric transducers working as an actuator and a sensor respectively. A damage is induced via reducing the elastic modulus of selected element by $50 \%$.

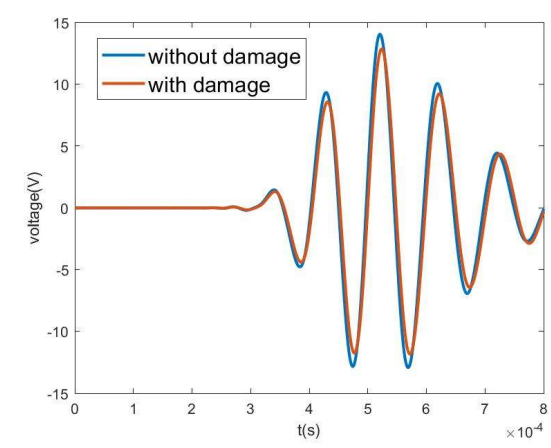

(a)

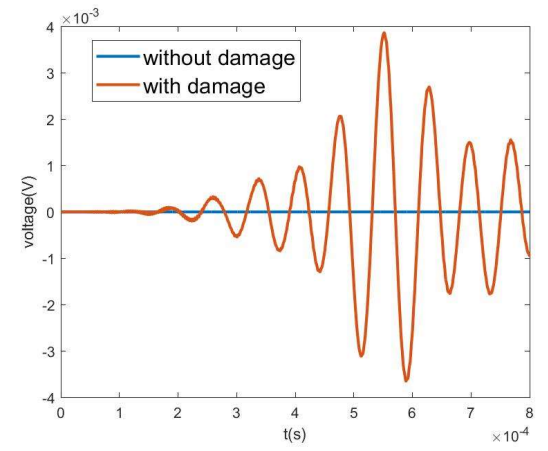

(b)

Figure 9 Comparison of voltage responses before and after damage existence of (a) sensor $L(0)$ and sensor $\mathbf{F}(6)$

Table 4 Magnitude of the voltage responses without damage

\begin{tabular}{c|c|c|c|c}
\hline & Sensor L(0) & Sensor T(0) & Sensor F(6) & Sensor F(4) \\
\hline Actuator L(0) & $\mathbf{1 0}^{\mathbf{1}}$ & $\mathbf{1 0}^{-9}$ & $\mathbf{1 0}^{-10}$ & $\mathbf{1 0}^{-9}$ \\
Actuator T(0) & $\mathbf{1 0}^{-9}$ & $\mathbf{1 0}^{-2}$ & $\mathbf{1 0}^{-9}$ & $\mathbf{1 0}^{-9}$ \\
Actuator F(6) & $10^{-9}$ & $\mathbf{1 0}^{-8}$ & $\mathbf{1 0}^{-9}$ & $\mathbf{1 0}^{-9}$ \\
Actuator F(4) & $10^{-9}$ & $\mathbf{1 0}^{-8}$ & $\mathbf{1 0}^{-9}$ & $\mathbf{1 0}^{\mathbf{0}}$ \\
\hline
\end{tabular}

Table 5 Magnitude of the voltage responses with damage

\begin{tabular}{|c|c|c|c|c|}
\hline 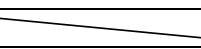 & Sensor L(0) & Sensor T(0) & Sensor F(6) & Sensor F(4) \\
\hline Actuator L(0) & $10^{1}$ & $10^{-7}$ & $10^{-3}$ & $10^{-9}$ \\
\hline Actuator T(0) & $10^{-8}$ & $10^{-2}$ & $10^{-9}$ & $10^{-4}$ \\
\hline Actuator F(6) & $10^{-1}$ & $10^{-8}$ & $10^{0}$ & $10^{-8}$ \\
\hline Actuator F(4) & $10^{-8}$ & $10^{-3}$ & $10^{-8}$ & $10^{0}$ \\
\hline
\end{tabular}

$\mathrm{L}(0), \mathrm{T}(0), \mathrm{F}(6)$, and $\mathrm{F}(4)$ are $12.5 \mathrm{khz}, 7.95 \mathrm{khz}, 1.13 \mathrm{khz}$, and $1.36 \mathrm{khz}$ respectively. Table 4 lists the voltage magnitudes of all 16 cases for the undamaged situation. The diagonal numbers are several magnitudes higher than the non-diagonal elements. This is coincided with the aforementioned analysis.

Later a damage, or rather an asymmetry, is induced via reducing the elastic modulus of selected element by $50 \%$. When wave $\mathrm{L}(0)$ is actuated buy the actuator, voltage responses of sensors $\mathrm{L}(0)$ and $\mathrm{F}(6)$ are presented in Figure 9. Compared to the undamaged cases, voltage response of $\mathrm{L}(0)$ reduces with a small extent while voltage response of $\mathrm{F}(6)$ increases from $10^{-10}$ to $10^{-3} \mathrm{~V}$. That is, a part of lost energy in $\mathrm{L}(0)$ is transferred to $\mathrm{F}(6)$ due to interaction with damage. Results of all 16 cases are listed in Table 5. Wave mode conversion between $\mathrm{L}(0)-\mathrm{F}(6)$ and $\mathrm{T}(0)-\mathrm{F}(4)$ are clearly observed.

All these conducted numerical simulations demonstrated that the designed wave transducers are capable to interact with only one wave with high sensitivity. This selective electromechanical coupling feature can be used to detect small amount of wave mode conversion due to local damage.

\section{Monitoring the connection status of a flange}

Eventually we apply these actuators to monitor the connection status of a flange, which is widely used in aeronautic and astronautic structure systems. The flange is modelled by Equivalent thin layer method(Yao, Wang and Zhai, 2015) as shown in Figure 10. The basic idea is to use an elastic media as the replacement of the joint structure.

Two kinds of damage are simulated by changing elastic modulus of local element in $30^{\circ}$ range and angle of damaged element respectively, as shown in Figure 11. The aim is to simulate the loosening of one or three bolts.

The axial length of flange is $0.02 \mathrm{~m}$ and consequently working frequencies for actuator $\mathrm{L}(0), \mathrm{T}(0), \mathrm{F}(6)$ and $\mathrm{F}(4)$ are $132.70 \mathrm{khz}, 78.51 \mathrm{khz}, 120.14 \mathrm{khz}$, and $120.24 \mathrm{khz}$. The result of actuator $\mathrm{L}(0)$ is partly shown in Figure 14 . With increase of elastic modulus of whole joint structure, wave mode transmission can be observed in Figure 12(a)(b) that voltage 


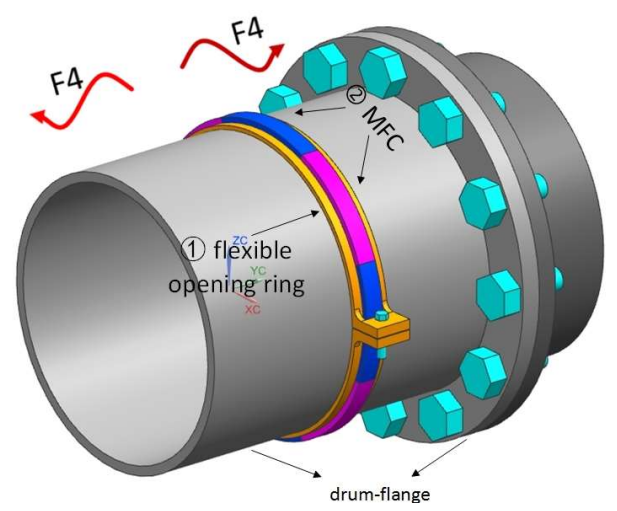

(a)

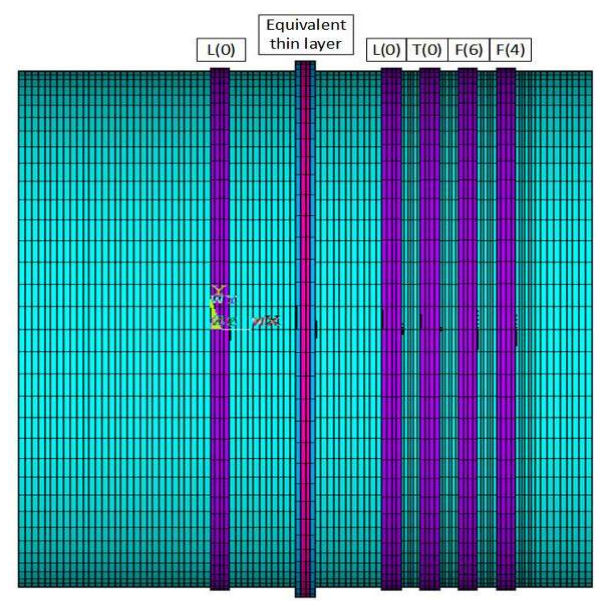

(b)

Figure 10 Drum-flange: the left is actuator $L(0)$ and the right are sensor $L(0), T(0), F(6)$ and $F(4)$

of sensor L(0) monotonously decreases and meanwhile its signal energy illustrates negative correlation with increased elastic modulus. Furthermore, voltage of sensor $\mathrm{T}(0), \mathrm{F}(6)$, and $\mathrm{F}(4)$ are $10^{-9}, 10^{-10}$, and $10^{-10} \mathrm{~V}$ accorded with conclusion in section 3.2 due to inexistence of asymmetry. Wave mode transmission is consequently suggested for inverse identification of dynamic parameters of whole joint structure.

When two kinds of damage are induced, minor changes in Figure 12(c)(d) indicate restriction of wave mode transmission that local damage is invisible. On the contrary, wave mode conversion between $\mathrm{L}(0)-\mathrm{F}(6)$ shows high sensitive for local damage. As shown in Figure 12(e)(f), when elastic modulus of local damage increase, voltage and its signal energy of sensor $F(6)$ decrease demonstrating a negative correlation. Besides, in Figure $12(\mathrm{~g})(\mathrm{h})$, when angle of damage element is changed by every $15^{\circ}$ from $0^{\circ}$ to $90^{\circ}$, voltage and its signal energy of sensor $F(6)$ have symmetry about $30^{\circ}$ and $60^{\circ}$. These two kinds of damage are accurately detected, and inverse damage qualification can be conducted due to this quantitative work.

\section{Conclusions}

In this paper, we proposed a design scheme of distributed piezoelectric transducers in cylindrical shell and utilized them

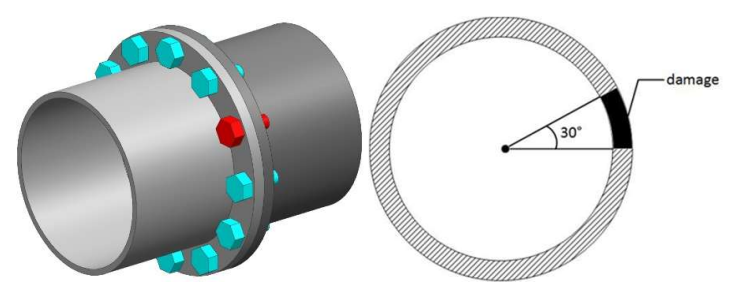

(a)

(b)

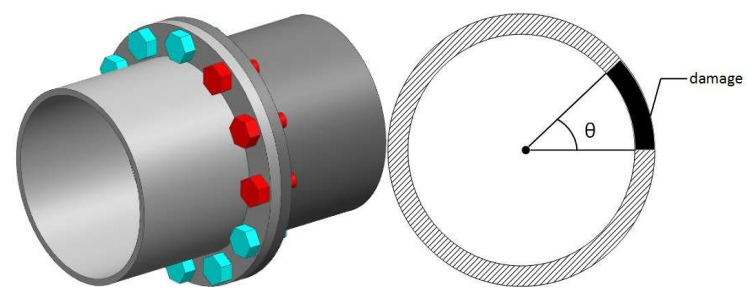

(c)

(d)

Figure 11 Two features (a)(c) of damage in flange and their simulations (b)(c). The red one is damaged bolt and the blue one is undamaged

to detect the scattering characteristics of waves induced by the status changes or damages of the joint structures.

The main conclusions are as follows:

(a). Distributed function and polarization direction of transducers was designed to cancel the charge contributed from the undesired waves in cylinder. This was based on orthogonality between wave modes. Simulation results showed ten orders of magnitude difference in voltages between targeting wave mode and other wave modes. It was demonstrated the transducer is only coupled with target wave mode.

(b). With a damage/asymmetry (\%50 loss of elastic modulus) induced in homogeneous shell structure, the voltage of $\mathrm{L}(0)-\mathrm{F}(6)$ increased from $10^{-9} \mathrm{~V}$ to $10^{-1} \mathrm{~V}$. That is, asymmetry can cause wave mode conversion in cylinder and this can be detected by transducers.

(c). By increasing elastic modulus of equivalent thin layer of flange structure, the decrease of voltage of wave mode transmission suggested that the transmission can be used for dynamic parameter identification. Nevertheless, when two types of local damage induced, the voltage of wave mode transmission almost unchanged while wave mode conversion showed high sensitivity to local damage and had dramatically symmetry about angle of damage.

In this work, the scattering characteristic of waves in cylinder showed a strong relationship with connection status of flange. All these conducted numerical simulations demonstrated that the designed wave transducers are very promising for the SHM of joint structures. Future work includes experiment to verify the performance of transducers.

\section{Acknowledgment}

The authors would like to acknowledge the support of National Natural Science Foundation of China [Grant Nos. 51675022 and 11702011]. 


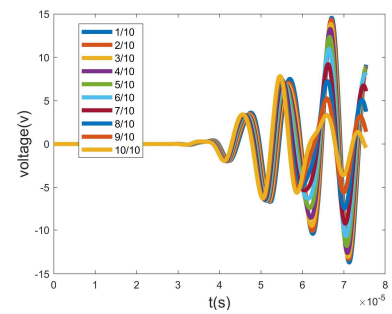

(a)

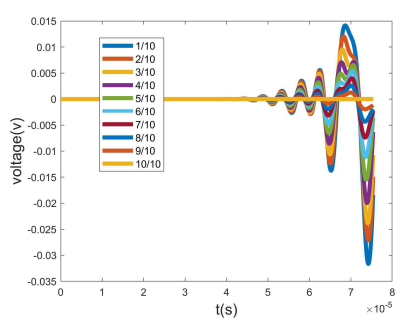

(e)

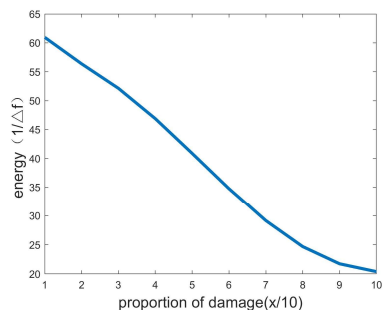

(b)

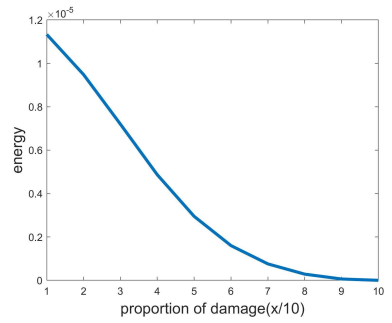

(f)

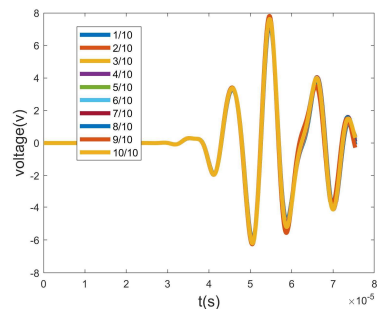

(c)

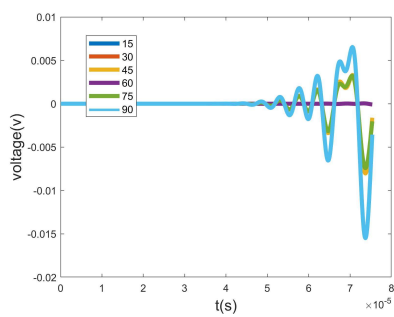

(g)

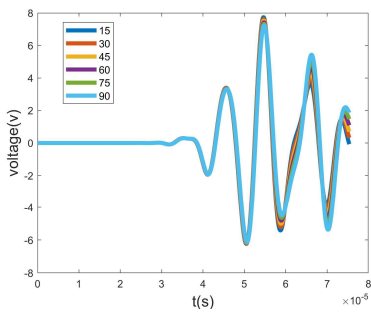

(d)

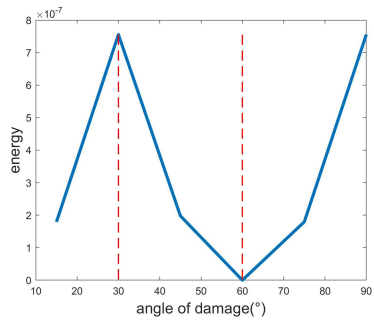

(h)

Figure 12 (a) Voltage and (b) its energy of sensor $L(0)$ with increase of elastic modulus of whole joint structure. Voltage of sensor $L(0)$ with two features of damage(c)(d). (e) Voltage and (f) its energy of sensor $F(6)$ with first feature of damage. $(g)$ Voltage and $(h)$ its energy of sensor $F(6)$ with second feature of damage.

\section{References}

Arthur W. Leissa - Vibration of Shells (1993). Acoustical Society of Amer.

Deraemaeker, A. et al. (2010) 'New Trends in Vibration Based Structural Health Monitoring', International Centre for Mechanical Sciences, (520), pp. 1-643.

Farrar, C. R. et al. (2009) 'A low-power wireless sensing device for remote inspection of bolted joints', Proceedings of the Institution of Mechanical Engineers, Part G: Journal of Aerospace Engineering, 223(5), pp. 565-575.

Fleming, A. J. (2006) Piezoelectric Transducers for Vibration Control and Damping, Piezoelectric Transducers for Vibration Control and Damping.

Fromme, P. (2004) 'On the Scattering and Mode Conversion of the A0 Lamb Wave Mode at Circular Defects in Plates', 142(April), pp. 142-149.

Furusawa, A., Kojima, F. and Morikawa, A. (2015) 'Mode control of guided wave in magnetic hollow cylinder using electromagnetic acoustic transducer array', Nuclear Engineering and Technology. Elsevier B.V, 47(2), pp. 196203.

Hamzeloo, S. R., Shamshirsaz, M. and Rezaei, S. M. (2012) 'Damage detection on hollow cylinders by ElectroMechanical Impedance method: Experiments and Finite Element Modeling', Comptes Rendus - Mecanique. Elsevier Masson SAS, 340(9), pp. 668-677.

Kharrat, M. et al. (2014) 'Pipeline inspection using a torsional guided-waves inspection system. Part 1: Defect identification', International Journal of Applied Mechanics, 6(4).

Lee, B. C. and Staszewski, W. J. (2003) 'Modelling of Lamb waves for damage detection in metallic structures: Part II. Wave interactions with damage', Smart Materials and Structures, 12(5), pp. 815-824.
Li, F. et al. (2015) 'Guided wave propagation in high-speed train axle and damage detection based on wave mode conversion', Structural Control and Health Monitoring, 22(9), pp. 1133-1147.

M Maia, N. M. (2006) 'Shock and Vibration Digest A REVIEW ON VIBRATION-BASED STRUCTURAL HEALTH MONITORING WITH SPECIAL EMPHASIS ON COMPOSITE MATERIALS'. Available at: http://eprints.bournemouth.ac.uk/24581/1/2006 Montalvao Review SHM Composites Final SVD-05-06 PRE-PRINT.pdf. Na, W. S. and Baek, J. (2018) 'A review of the piezoelectric electromechanical impedance based structural health monitoring technique for engineering structures', Sensors (Switzerland), 18(5).

Nilsson, A. and Liu, B. (2015) Vibro-Acoustics, Volume 2, Vibro-Acoustics, Volume 1.

Peairs, D. M., Park, G. and Inman, D. J. (2004) 'Practical issues of activating self-repairing bolted joints', Smart Materials and Structures, 13(6), pp. 1414-1423.

Willberg, C. et al. (2012) 'Continuous mode conversion of Lamb waves in CFRP plates', Smart Materials and Structures, 21(7).

Willberg, C. et al. (2015) 'Simulation Methods for Guided Wave-Based Structural Health Monitoring: A Review', Applied Mechanics Reviews, 67(1), p. 010803.

Yao, X. Y., Wang, J. J. and Zhai, X. (2015) 'Modeling method of bolted joints of aero-engine based on thin-layer element', Journal of Beijing University of Aeronantics and Astronantics, 41(12), pp. 2269-2279. 